\title{
KABUKI-MONO: The Art of Kumadori Facial Expression for Manga and Cosplay
}

$\operatorname{AUTHOR}(\mathrm{S}):$

Tosa, Naoko; Konoike, Ryotaro; Nakatsu, Ryohei

\section{CITATION:}

Tosa, Naoko ...[et al]. KABUKI-MONO: The Art of Kumadori Facial Expression for Manga and Cosplay. 2011 International Conference on Culture and Computing 2011: 98-103

\section{ISSUE DATE:}

2011

URL:

http://hdl.handle.net/2433/250228

\section{RIGHT:}

(C) 2011 IEEE. Personal use of this material is permitted. Permission from IEEE must be obtained for all other uses, in any current or future media, including reprinting/republishing this material for advertising or promotional purposes,

creating new collective works, for resale or redistribution to servers or lists, or reuse of any copyrighted component of this work in other works.; This is not the published version. Please cite only the published version.; この論文は出版社版

でありません。引用の際には出版社版をご確認ご利用ください。 


\section{KABUKI-MONO}

\section{The Art of Kumadori Facial expression for Manga and Cosplay}

\author{
Naoko Tosa \\ Academic Center for Computing \\ and Media Studies \\ Kyoto University \\ Kyoto, Japan \\ e-mail:tosa@media.kyoto-u.ac.jp
}

\author{
Ryotaro Konoike \\ Department of engineering \\ Kyoto University \\ Kyoto, Japan \\ e-mail:konoike@qoe.kuee.kyoto- \\ u.ac.jp
}

\author{
Ryohei Nakatsu \\ Interactive \& Digital Media Institute \\ National University of Singapore \\ Singapore \\ e-mail: idmdir@nus.edu.sg
}

\begin{abstract}
An idea in the "Art of Cultural Computing" is that any type of local culture could be accepted globally. This work encourages people to appeal their cultures widely. The Web connects people far all over the world. From the integration of various cultures in the world, new pop culture emerges. One example is that young generation across the world enjoy Japanese Manga (comic) and Cosplay (costume-play). These Japanese cultures fulfill a human desire to become someone else. Japan has various other cultural activities: one is "Kabuki," born in the Edo era and enjoyed by many people since then. The term Kabuki is from "Kabuku" which means "out of the ordinary." Kabuki actors used to make up their faces in a special way, speak exaggeratedly, and over-act, thus appealing to audience. These special behaviors and make up are similar to punk fashion. The makeup, called Kumadori, is like makeup of native Africans and native Americans. These come from a desire to appeal to others. This means that is important to appeal our local culture to the world and share it among people all over the world. That will form a basis of new global culture to understand each other and evolve through mutual integration.
\end{abstract}

Keywords-Media Art, JapanesePOPculture, Manga,Cosplay,

\section{INTRODUCTION}

Recently, many local cultures are glowing their popularities in the global society by creating various new pop cultures. For example, Japanese Manga (comic) or Anime (animation) are now fascinating young people all around the world [1][2][3]. The characters in Manga are being their heroes or heroines. Readers of Manga created a bland new culture called Cosplay (costume play) that means they dress up as same as the characters in their favorable Manga. Cos-play is like Japanese Kabuki culture in some way.

In this paper we focused Kumadori, which is the special way of make-up in Kabuki, with the aim to create a new artwork that connects recent pop culture such as Cosplay and Japanese traditional culture. The basic design of Kumadori has been developed utilizing Buddhism statues' strong expression of muscles and refined facial expressions as well as those of Noh masks. The blood vessels and muscles are drawn on a player's face to exaggerate his emotion. A player with different roles is made up with different Kumadori design.
"Sujikuma," that means stormy sanguine character, has bold and sharp lines and "Ippon-kuma," that has calmer strong lines, is used for young hero who has great sense of justice. Awesome handsome guy uses "Mukimi" make up that draws sanguine line under the lids.

Black or indigo Kumadori is for cruel and ghostly characters like "Kuge-are" for a man who attempts to do subversion of a nation. "Hannya-kuma" for a woman who turns into an evil of jealousy. "Kijo-kuma" for a female specter and "Bohrei-kuma" for a ghost with stormy grudges. Blown colored Kumadoris are for sly and fox, or cruel characters like "Tuchi-gumo." We have investigated the relation between these Kumarodi make-ups and emotions conveyed by them and developes a feeling map of Kumadori. Fig.1 shows the Kumadori feeling map we have developed.

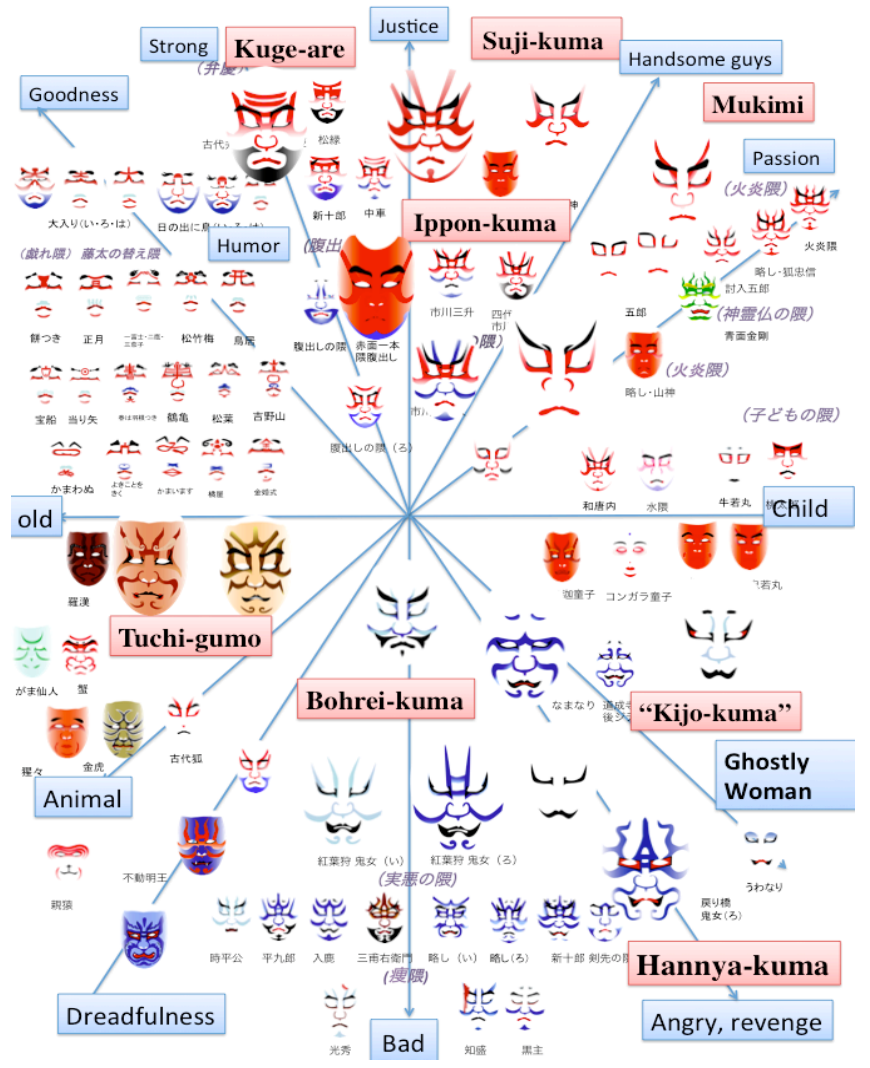

Figure 1. Kumadori feeling map 
Though Kumadori is one of Japanese make-ups, there are some common characteristics among Kumadori and makeups of Native Americans or native Africans. We propose a "Cosplay" software for digital cameras that connects Kabuki Kumadori with people in different culture, with a hope to appeal beauty of Japanese. We have developed a system that enables worldwide users to take pictures where Kumadoris are mapped on their faces. Also, the system enables the user to input any sentences on the speech balloon in the picture.

\section{STRUCTURE OF KABUKI-MONO SYSTEM: \\ COMPOSITE EFFECT OF FACE AND KABUKI MAKE-UP}

Composite system of facial image and Kabuki make-up creates a composite image that consists of a face image with Kabuki make-ups, by overlapping a Kumarodi pattern on face image, a wig for Kabuki, a background pattern, and an image of speech balloon. One of author development: the Virtual Kabuki system. It was a computer vision system estimates facial expressions and body postures and reproduces them in a computer graphics avatar in real time [4]. We used OpenCV to detect facial parts from an input photo, and then we developed an automated algorithm by adding several functions. We also developed a program that automatically detects the key positions on face where Kumadori pattern is overlapped as well as the wig image. The brief processing flow of this system is described below:

(1) Automatic recognition of facial parts and facial edges (OpenCV + Original method) Figure 2.
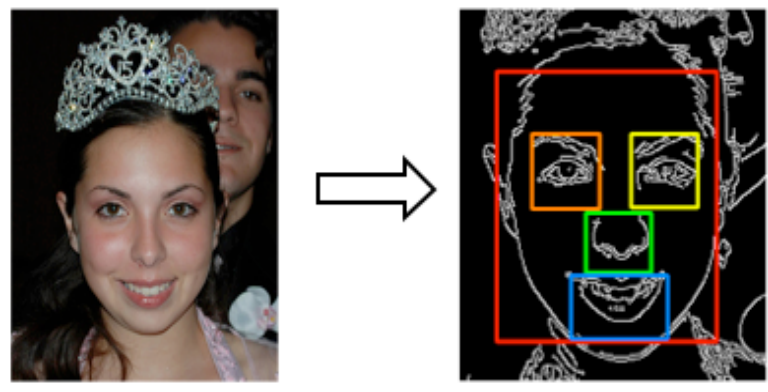

Figure 2. Result of recognition

(2) Direct transformation of the Kumadori image to fit to the face. Figure 3.
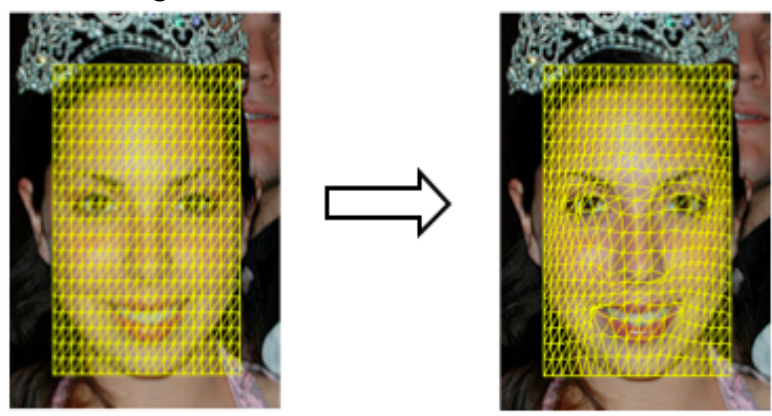

Figure 3. Result of direct transformation
(3) Clipping of the region of a face, and overlap of background pattern images. Figure 4 .

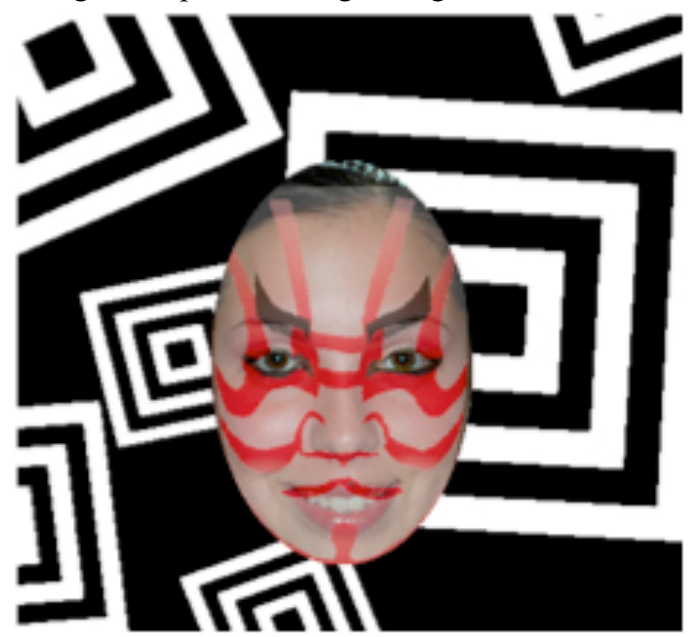

Figure 4. Result of cripping and overlap of Kabuki pettern

(4) Overlap of Kabuki-wig on the photo

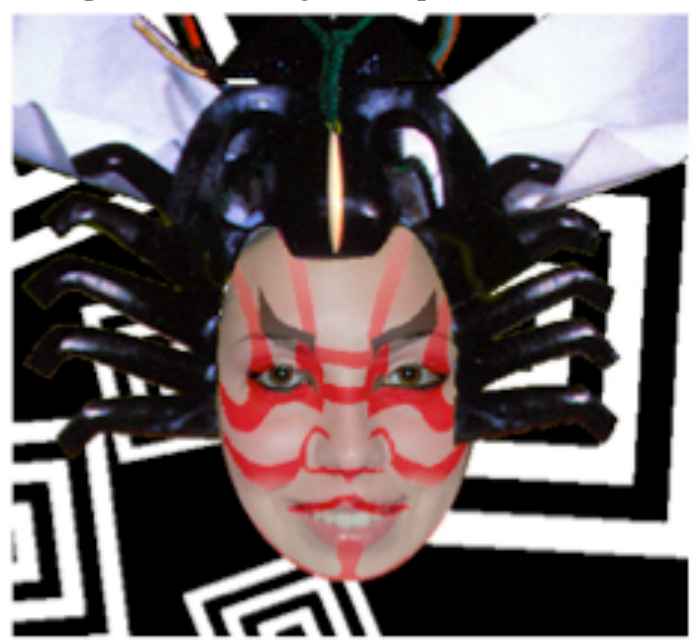

Figure 5. Result of systhesis ofKabuki-wig.

(5) Overlap of the speech balloon with user comment on the photo

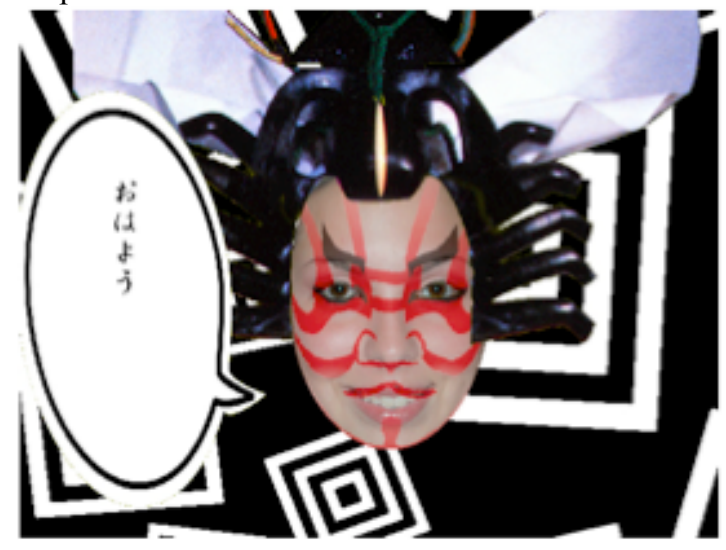

Figure 6. Result of synthesis of speech balloon with comment "Hello" 


\section{AUTOMATIC DETECTION OF THE FACIAL PARTS}

OpenCV has components to detect faces, eyes, noses or mouths in the picture. The results are accurate, but are returned in the forms of rectangle images. So we can't point out the feature points of eyes, mouths and so on. This means we should detect feature points from the rectangle image returned by OpenCV. We have developed an original method to detect these feature points of edges, mouths and eyes.

We have developed a method to detect feature points (red colored points) of facial parts within a detected rectangle Fig.7 (left). There are small mismatches to be corrected between the image and the detected feature points. We have developed particular methods each of which is dedicated to eyes, mouth, nose, and facial edges in order to correct the feature points. A result is shown in Fig.7 (right). We can see that feature points are rightly corrected. The correction process will be described below.

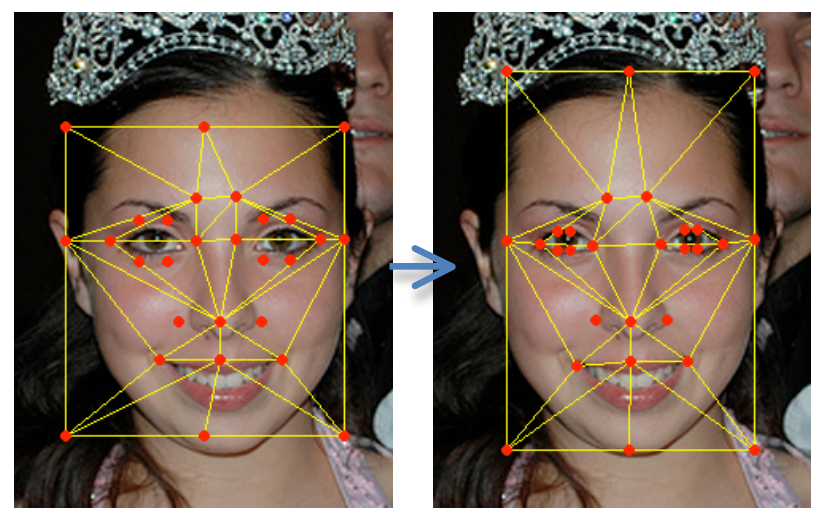

Figure 7. Automatic preparation of points and Corrected feature points

\section{A. Correction of the feature points of a mouth}

Fig.8 (left) shows an example of the incorrectly detected feature points of a mouth (the points are, left-edge, center and right-edge). Fig. 8 (right) shows a result of the correction process. Fig. 9 shows the detected edges of a mouth. This system utilizes vertically and horizontally scanned histograms to detect the feature points of a mouth by the density. A gray-colored region in Fig.4 shows the vertical and horizontal histograms. The system detects drastic rise in density from 0 (red dashed lines) as the edge lines. Then white pixels on the edge lines (yellow star marks) are assumed as feature points.
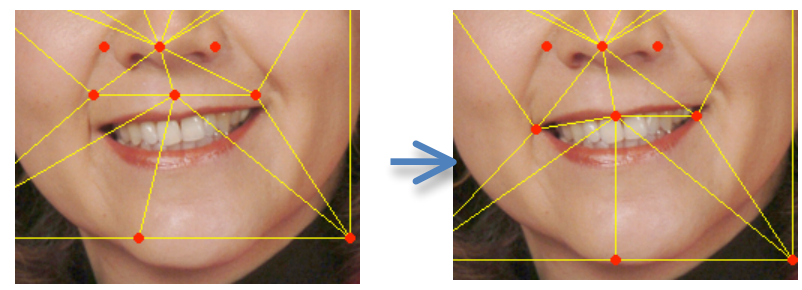

Figure 8. Without correction and With correction

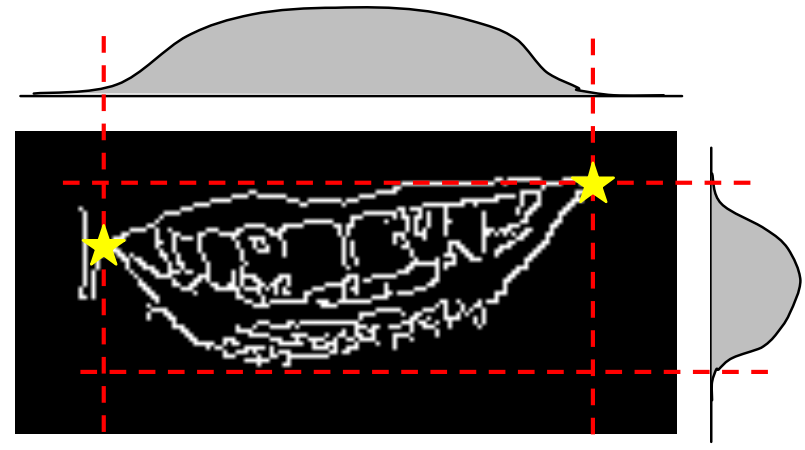

Figure 9. Edge image and histogram of mouth

\section{B. Correction of the feature points of eyes and a nose}

In correcting the feature points of eyes, the system makes histograms as I explained in previous section. After that, the system scans on the red dashed line shown in fig. 10 to detect maximum and minimum values of white pixel positions. The feature points are corresponding to upper and lower lids.

Correction of the nose feature points is almost the same as that of mouths or eyes. The system detects each edges (red dashed lines) to detect feature points. It showed Figure 11 .

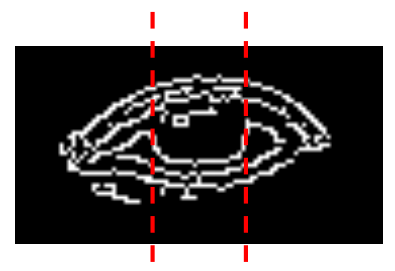

Figure 10. Positions of upper/lower lids

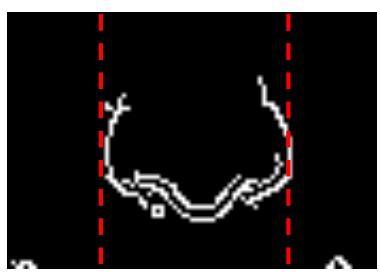

Figure 11. Position of Nose

\section{Correction of the feature points of facial edges}

Fig.12 shows the incorrectly detected feature points of facial edges. There are gaps in left and right image especially in upper/lower side of the rectangle. In order to overlap a Kumadori image on the face, we need to correct these mismatches. This system uses labeling method to detect faces (blue region in fig.12) and detects outside edges. Also, this system detects edges from original photo (fig.10) 
to compare each edge to determine final facial edges based on the similarity between two edges.

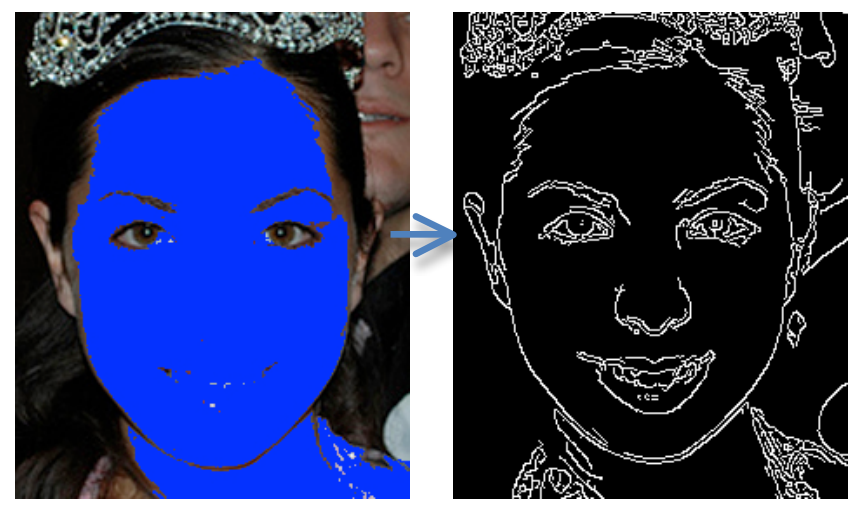

Figure 12. Labeled image of a face and Edge image of a face

\section{TRANSFORMATION OF KUMADORI IMAGES}

Fig.13 shows an example of a Kumadori image. This system transforms this image to mix on the given face.

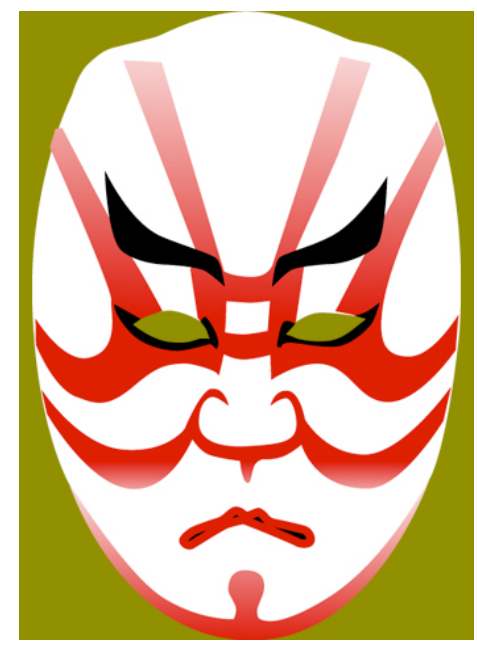

Figure 13. Kumadori image

Kumadori image has transparent regions for eyes, like masks. We need to match each facial part with the Kumadori make-up. This system uses feature points of each facial part to transform the Kumadori image to match correctly to the face. Fig.15 (left) shows all the feature points in red circles. We have selected 28 feature points to sufficiently represent the feature of the face. Fig.15 (right) shows a template image of Kumadori marked by green star as feature points. The feature points of both images are corresponding with each other. After the feature points of a facial image are corrected, the Kumadori image is transformed to match the feature points.
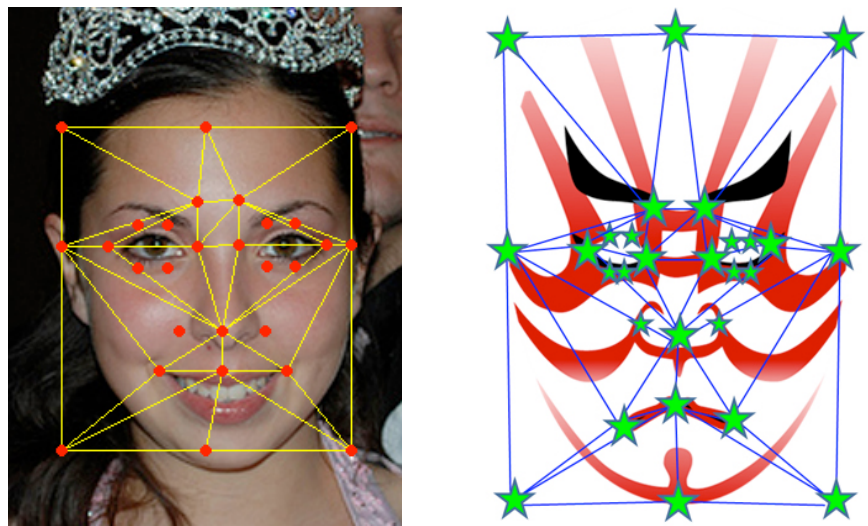

Figure 14. Feature points of a face and Feature points of a Kumadori

The transformation of a Kumadori image is done by splitting the image into mesh structure to calculate the moving distance of each cells. Vertices that are near to feature points moves in large step, or vice versa. Fig.15 shows the mesh structure (triangle polygons) and feature points (red triangle mark). Right figure shows the transformation of mesh when we moved a feature point to right-top direction. The system uses those methods to obtain smooth synthesis of Kumadori image on a face. Figure 17 is transformation of mesh with face.
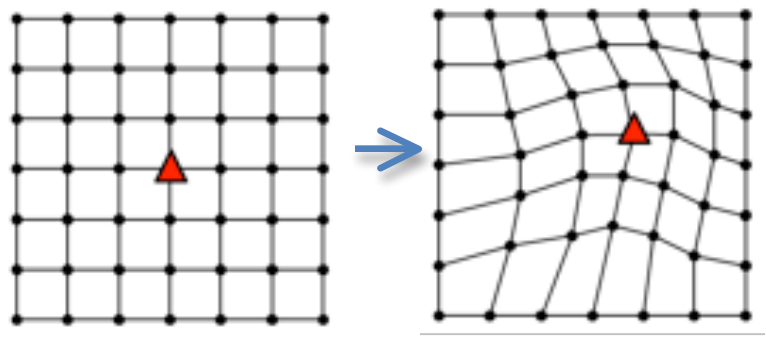

Figure 15. Transformation of mesh

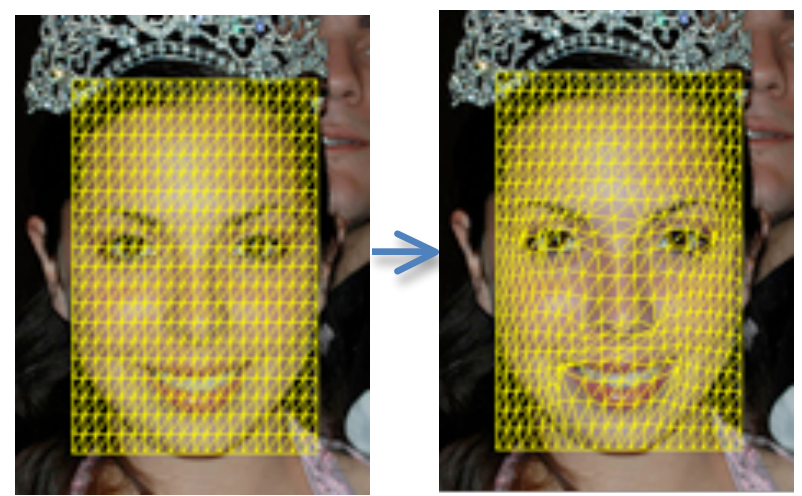

Figure 16. Before transformation of mesh and After transformation of with mesh 


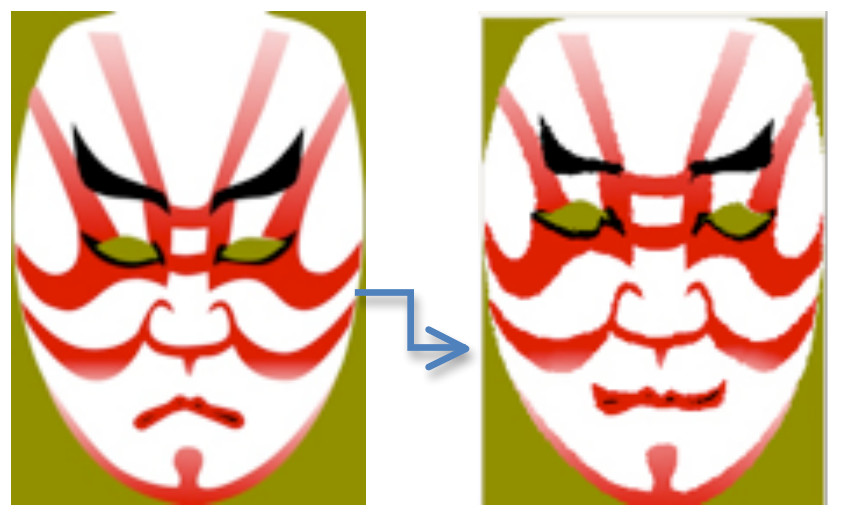

Figure 17. Before transformation of Kumadori image and After transformation of Kumadori image

By introducing this method it would become possible to change the original photo by changing the mesh pattern of the image. Even in this case it would be easy to overlap Kumadori pattern on a transformed image. An example of such facial image transformation is shown in Fig. 16 and Kumadori image transformation is shown in Fig.17.

\section{RESULTS}

Figure 12 shows the input photo, and Fig.13 shows the composite image of Kumadori, and Fig.14 shows the result of this system, mixed with a pattern background pattern and a speech balloon. The purpose of the speech balloon is to emphasize the effect of the generated image. An user can input relevant word such as "Cool," "Cute," or relevant onomatopoeia such as "Baaan,"

We want you to see because it made the movie that puts the costume play doing and manga the speech balloon and makes shading off that suits the face of people all over the world and famous people in Europe and America, Latin America, Turkey, Africa, the Mediterranean sea, Asia, and Oceania. [5]

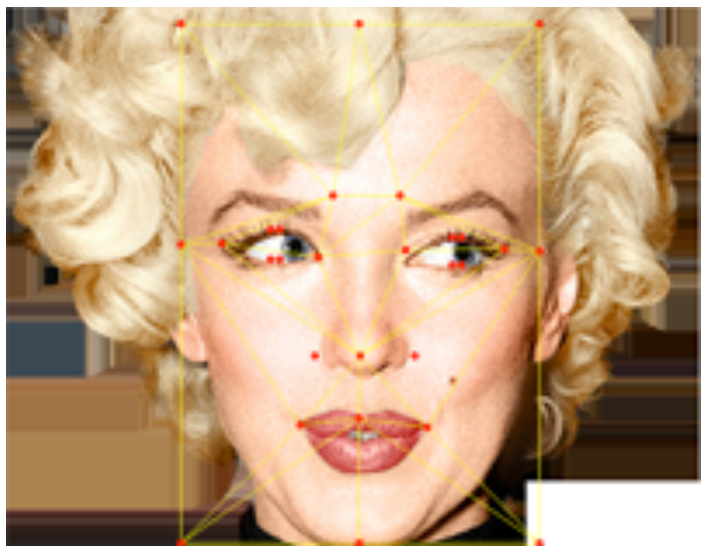

Figure 18. Facial detection

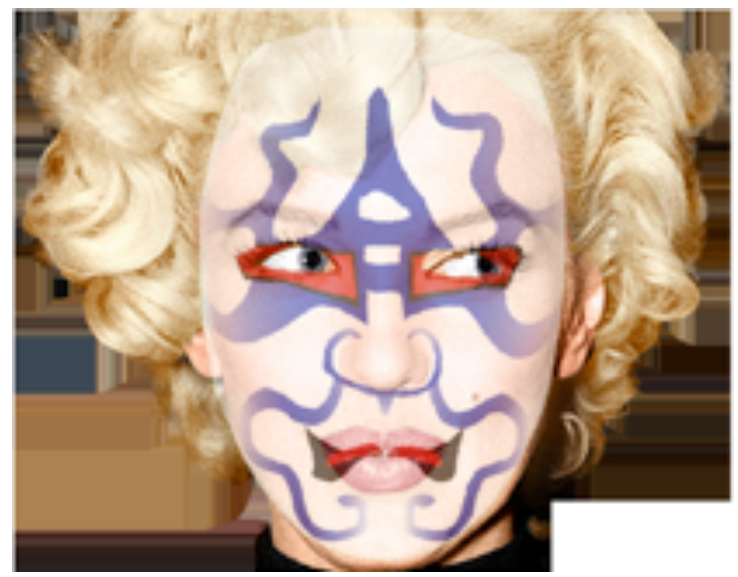

Figure 19. The composite image of the Kumadori

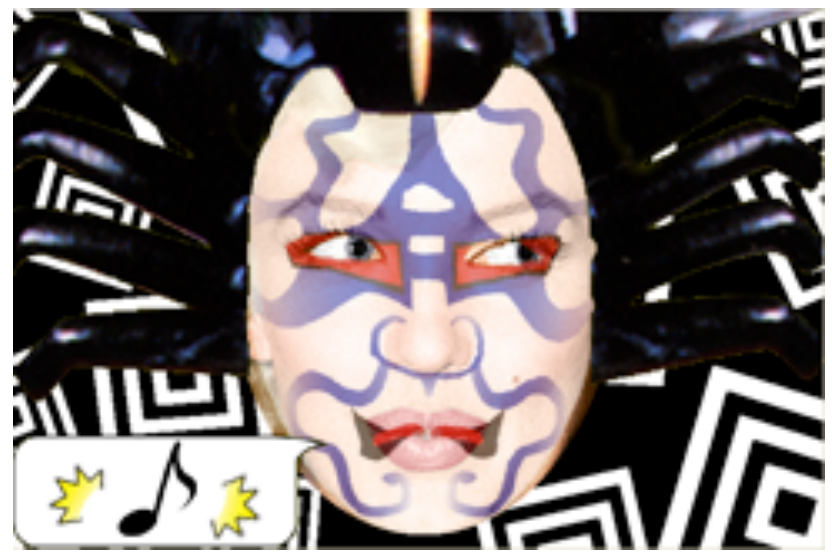

Figure 20. The result of this system, mixed with a Kabuki pattern background and a speech balloon of comment.

\section{CONCLUSION}

This collaborative research has been done with Nikon Co., Ltd. and Interactive \& Digital Media Institute National University of Singapore. At present stage this system doesn't have the function of automatically identifying the emotion of a face and selecting a relevant Kumadori pattern using the feeling map shown in Fig 1. The system allows the users to select the Kumadori in order to reflect their favors. The reason is that the user may not like automatically selected Kumadori. As an next step, we need to study the formation of such a complex cultural information.

We are going to carry out experiments to proof the effect of such a system as a web based photo album for digital cameras so that anybody can use the system.

As another direction of future work, we are looking for availabilities of new advanced system that generates cartoonlike image from an original image by exaggerating faces in the picture, like that a Kabuki player appears into Manga as one of the characters.

Such a research is a new application of a lot of digital archives in the world. There is only neither collection nor 
seeing the culture, and the simulation of the kabuki theater that not is tried actually by using a digital expression. [6] Using it to create the art exceeding a digital simulation will come to give a big impact to a modern art culture.

\section{Reference}

[1] Abbott, M., Visual representation of emotion in manga: LOSS OF CONTROL IS LOSS OF HANDS in Azumanga Daioh Volume 4 LANGUAGE AND LITERATURE (0963-9470) 2011.

Vol.20,No.2;p.91-112

[2] Deng, X.; Li, M.; Zhang, Y., "Research and development of the generation in Japanese manga based on frontal face image" 2010 IEEE 11th International Conference on Computer-Aided Industrial Design and Conceptual Design, CAID and CD'2010
[3] Nobuoka, J., "USER INNOVATION AND CREATIVE CONSUMPTION IN JAPANESE CULTURE INDUSTRIES: THE CASE OF AKIHABARA, TOKYO," GEOGRAFISKA ANNALER SERIES B-HUMAN GEOGRAPHY (0435-3684) 2010. Vol.92,No.3;p.205-218

[4] Ohya, J.; Kurumisawa, J.; Nakatsu, R.; Ebihara, K.; Iwasawa, S.; Harwood, D.; Horprasert, T. "Virtual metamorphosis," Multimedia, IEEE (1070-986X) 1999. Vol.6,No.2;p.29-39

[5] http://www.youtube.com/watch?v=-zwPPn0Lhpo

[6] Furukawa, K.; Akama, R.; Hirose, C.; Hachimura, K. "Digital Reconstruction of a Historical Kabuki Theater" Intelligent Information Hiding and Multimedia Signal Processing, 2009. IIHMSP '09. Fifth International Conference on (978-1-4244-4717-6) 2009. p.1160-1163 